\title{
Concept for the Calibration of the Rogowski Coil System of the Wendelstein 7-X Stellarator
}

\author{
Ulrich Neuner ${ }^{\mathrm{a}}$, Kian Rahbarnia ${ }^{\mathrm{a}}$, Michael Endler ${ }^{\mathrm{a}}$, Christoph von Sehren ${ }^{\mathrm{a}}$, Henning Thomsen ${ }^{\mathrm{a}}$, \\ Thomas Klinger ${ }^{\mathrm{a}}$
}

${ }^{a}$ Max-Planck-Institut für Plasmaphysik, Wendelsteinstr. 1, 17491 Greifswald, Germany

\begin{abstract}
While the low frequency part of the response of the Rogowski coils mounted in the stellarator Wendelstein 7-X has been calculated, the high frequency part is planned to be calibrated by means of an actively applied current. In a staged approach different conductors have been designed to be mounted inside the plasma vessel and an AC current of $1 \mathrm{kA}$ is foreseen to be driven through them at frequencies up to $1 \mathrm{kHz}$ to simulate the plasma current.
\end{abstract}

Keywords: Rogowski coils, calibration, diagnostics, current measurement.

\section{Motivation}

Twelve Rogowski coils [1] have been installed in the vacuum vessel of the stellarator Wendelstein $7-X$ (W7-X) [2-4] and have been commissioned [5] as part of the set of MHD diagnostics [6]. They are designed to measure the equilibrium plasma currents (PfirschSchlüter current and bootstrap current) as well as beam driven currents. The Rogowski coils are sufficiently sensitive to measure currents below $1 \mathrm{kA}$ with an absolute accuracy of 100 A [1]. Coils are mounted inside and outside of the plasma vessel. While the time resolution of the outside coils is restricted to $\sim 25 \mathrm{~ms}$, the inside coils can measure well above $1 \mathrm{kHz}$ [1]. It is planned to calibrate these coils using a conductor positioned inside the plasma vessel with an alternating current passing through it. The response of the coils will be measured and compared to calculations.

\section{Calculated low frequency conversion factors}

The Rogowski coils of W7-X are positioned to measure toroidal currents. Due to constraints in manufacturing and assembly they are divided into several segments. The outputs of these segments are either connected in series to measure the total output only (continuous coils) or they are measured individually (segmented coils). Individual signals can either be added to work similar to a continuous coil and yield the total current, or they can be individually evaluated to yield information on the current density distribution.

A first rough estimation of the conversion factors used to calculate the plasma current from the time integrated voltages of the Rogowski coils could be performed by calculating the output voltage $U$ from the rate of current change $\mathrm{d} I / \mathrm{d} t$ by inserting the design values of the winding area $A$ and the winding density $N / l$ into formula (1)

$$
U=-A \frac{N}{l} \mu_{0} \frac{\mathrm{dI}}{\mathrm{dt}}
$$

The values thus obtained are listed in tables 1 and 2 in the column "Ideal conversion factor".

Due to restrictions in the available installation space, gaps of different sizes are present in the rings of segments that make up a Rogowski coil. These gaps lead to responses that are individually reduced as compared to the response of an ideal Rogowski coil without gaps. The individual conversion factors must therefore be increased according to the gap sizes.

To calculate these individual conversion factors for each Rogowski coil the magnetic field at the measured position of the coils was calculated, employing BiotSavart's law assuming a current along the magnetic axis. Taking the portion of the field aligned with the direction of the coil segments and integrating along their length, the coil's response was calculated as a sum over the responses of the individual segments. In this way the gaps between the segments were properly accounted for. The results are listed in tables 1 and 2 in the column "Individual conversion factor".

Table 1. Calculated conversion factors of continuous Rogowski coils. The column "Position" refers to the numbers in Fig. 4.

\begin{tabular}{llll}
\hline Coil name & $\begin{array}{l}\text { Posi- } \\
\text { tion }\end{array}$ & $\begin{array}{l}\text { Ideal } \\
\text { conversion } \\
\text { factor } \\
\text { in } \mathrm{kA} / \mathrm{mVs}\end{array}$ & $\begin{array}{l}\text { Individual } \\
\text { conversion } \\
\text { factor } \\
\text { in } \mathrm{kA} / \mathrm{mVs}\end{array}$ \\
\hline QXR11CE001 & 1 & -1.6419 & -1.8019 \\
QXR11CE002 & 1 & -2.0523 & -2.2533 \\
QXR11CE003 & 1 & -1.5574 & -1.6067 \\
QXR11CE004 & 1 & -1.5574 & -1.6069 \\
QXR40CE003 & 2 & -1.5574 & -1.6116 \\
QXR40CE004 & 2 & -1.5574 & -1.6124
\end{tabular}

Table 2. Calculated conversion factors of segmented Rogowski coils. The column "Position" refers to the numbers in Fig. 4. 


\begin{tabular}{llll}
\hline Coil name & $\begin{array}{l}\text { Po- } \\
\text { si- } \\
\text { tion }\end{array}$ & $\begin{array}{l}\text { Ideal } \\
\text { conversion } \\
\text { factor } \\
\text { in kA/mVs }\end{array}$ & $\begin{array}{l}\text { Individual } \\
\text { conversion } \\
\text { factor } \\
\text { in } \mathrm{kA} / \mathrm{mVs}\end{array}$ \\
\hline QXO21CE111-181 & 3 & -1.6419 & -1.8880 \\
QXO21CE112-182 & 3 & -1.6419 & -1.8868 \\
QXO21CE113-183 & 3 & -1.5574 & -1.7689 \\
QXO21CE114-184 & 3 & -1.5574 & -1.7688 \\
QXO31CE113-183 & 4 & -1.5574 & -1.7816 \\
QXO31CE114-184 & 4 & -1.5574 & -1.7807
\end{tabular}

Employing the calculated individual conversion factors, the signals of different Rogowski coils can be compared (Fig. 1). Overall (i.e. for low frequencies) the signals match reasonably well. Explaining the differences (between $200 \mathrm{~ms}$ and $700 \mathrm{~ms}$ the relative difference is considerable, between $700 \mathrm{~ms}$ and $1250 \mathrm{~ms}$ the relative difference is lower, but still unacceptably large) is a work currently in progress. The differences for high frequencies at the end of the pulse are explained in section 3.

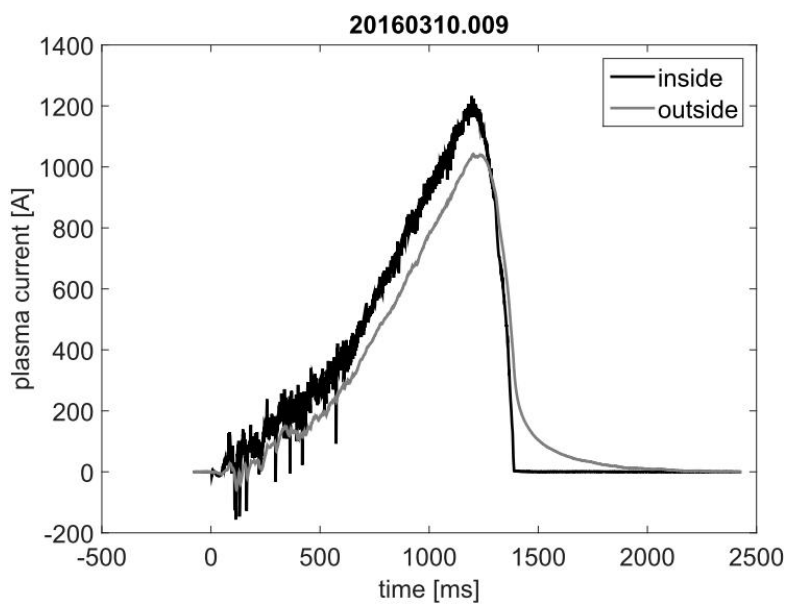

Fig. 1. Comparison of measurements with Rogowski coils QXR11CE002 mounted inside and QXR11CE004 mounted outside the plasma vessel during a W7-X plasma heated with $3.8 \mathrm{MW}$ ECRH. Both coils are in position 1 as indicated in figure 4 .

\section{High frequency dampening}

For high frequency components the signals in Fig. 1 are clearly mismatched. The signal from the outer coil seems to be a smoothed version of the signal from the inner coil.

With rising frequency the induced eddy currents in the plasma vessel wall (and to a lesser extent in other surrounding components, mainly first wall and divertor) affect the response of the Rogowski coils and dampen them. The coils on the outside of the plasma vessel wall (i.e. behind the wall) are much more affected by this than the coils on the inside of the plasma vessel wall (i.e. facing the plasma current).

Due to the complexity of the surrounding components this dampening by eddy currents will not be simulated by calculation, but instead it is planned to perform a calibration. This should also yield more precise results because of the considerable uncertainties in the knowledge of the electrical properties of the surrounding components.

The calibration of both inside and outside Rogowski coils will be done with an alternating current in a wide frequency range to cover the expected experimental output at W7-X. The upper limit of these frequencies will be $1 \mathrm{kHz}$. This is because above this frequency value the calibration factor of the Rogowski coils mounted on the inside of the plasma vessel is not expected to change until they reach their cut-off frequency, and the response of the coils mounted outside the vessel is expected to be too low to carry any useful information.

This calibration should yield a curve of the calibration factor as a function of signal frequency for each Rogowski coil. This will be applied to infer the plasma current from the measured signals. It will also yield the upper frequency limit of the Rogowski coils mounted on the outside of the plasma vessel, above which no meaningful information is to be expected from them.

To simplify procedures and to avoid unnecessarily lengthening the time needed for the calibration (during which all other work in the plasma vessel will be suspended), the signals of all Rogowski coils will be recorded during any calibration run.

\section{Conductor inside the plasma vessel}

At staged approach is envisioned for the actual calibration procedure.

\subsection{Single rail system}

The single rail system (Fig. 2) is a rail mounted temporarily inside the plasma vessel, spanning its whole circumference. In each cross section it is approximately in the center. Its main advantage is that no additional assembly time inside the plasma vessel of $\mathrm{W} 7-\mathrm{X}$ is needed. At the time of the calibration, the rail will be already installed inside the plasma vessel as a track for the neutron source that is employed for the obligatory calibration of the neutron detectors of W7-X. 


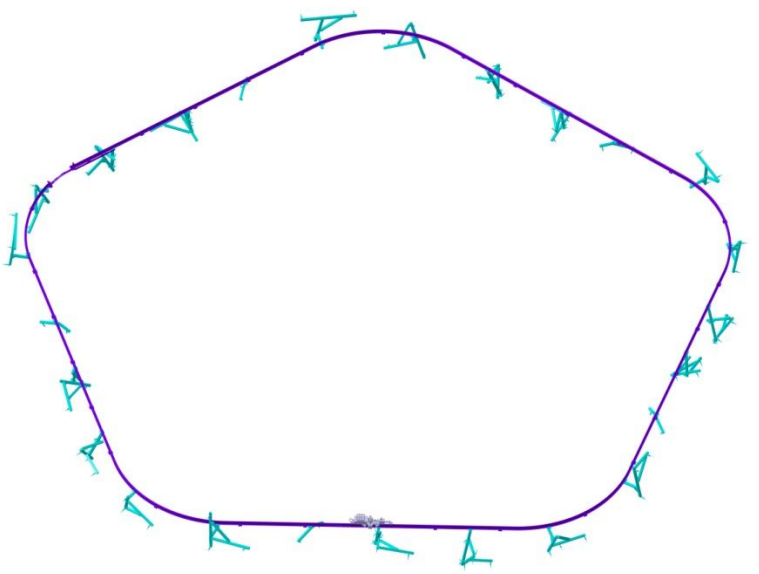

Fig. 2. Single rail system (slightly tilted view from the top), diameter approx. $10 \mathrm{~m}$. The rail is shown in violet, the supports that hold the rail in place are shown in light blue

The single rail system will be employed for the calibration of the continuous Rogowski coils (for the calibration of the segmented Rogowski coils see subsections 4.2 and 4.3). It has been already installed once in the plasma vessel for the neutron calibration preceding the first operational phase of $\mathrm{W} 7-\mathrm{X}$ and is ready to be installed again for both the neutron and the Rogowski calibration preceding the next operational phase of W7-X.

\subsection{Four conductor segment}

While the single rail system works well for the continuous Rogowski coils where only the total current needs to be known and the localization of the current does not affect the calibration, the segmented Rogowski coils need a well defined current localization since they are designed to measure current density distributions.

It is foreseen to optionally install - if assembly time permits - a single segment (Fig. 3) of the later to be installed four conductor system (see subsection 4.3). In this way, one of the segmented Rogowski coils could be calibrated. Since the location of the segment will be in module 4 of W7-X, this will be the Rogowski coil consisting of the two half-coils QXR40CE003 and QXR40CE004 in position 2 in figure 4.

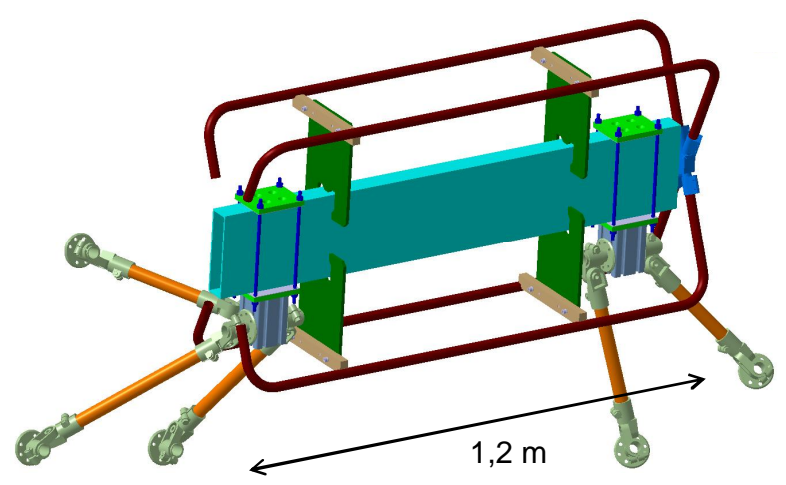

Fig. 3. Four conductor segment. The four copper rod conductors are shown in red, all other components belong to the support structure that holds the conductors in place.

The increase in the precision to which the current distribution is known (as compared to the single rail system) is achieved by two means: Firstly, in the four conductor segment the current is restricted to conductors that have a much smaller cross section than the single rail and secondly the four conductor segment is stiffer than the single rail so that its position can be measured to a higher precision.

The components of the four conductor segment have been manufactured and are currently being prepared for the later installation into the plasma vessel.

\subsection{Circumferential four conductor system}

It is foreseen to install at a later time a dedicated multi-loop system (Fig. 4). It will be mounted in those four of the five modules of W7-X where Rogowski coils are located. In each cross section it is approximately in the center.

The time needed to assemble and disassemble this system inside the plasma vessel will be considerable, so that a long shut-down period of W7-X without too much other in-vessel work is needed.

This system will be employed to calibrate all segmented Rogowski coils. It will yield a well defined localization of the current as described in subsection 4.2 for a single segment and the possibility to vary the position of the current for more detailed studies of the response of the Rogowski coils to different current density distributions.

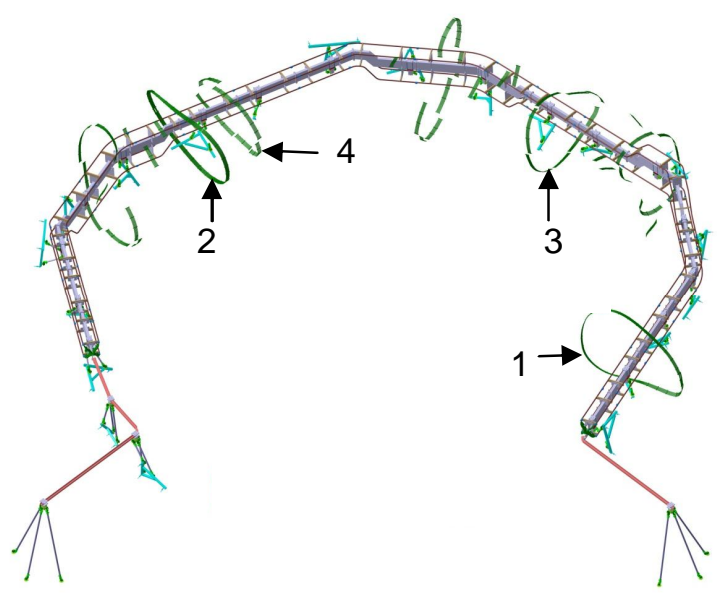

Fig. 4. Circumferential four conductor system, diameter approx. $10 \mathrm{~m}$, shown together with the Rogowski coils. The numbers in the figure refer to the positions of the Rogowski coils as listed in tables 1 and 2. The Rogowski coils are shown in green, the four conductor system in grey. Its internal design is that of the four conductor segment shown in fig. 3 , it is simply longer and curved. Supports are shown in light blue and the components to the lower left and right are current feeds. 
The design of this circumferential four conductor system has been finished.

\section{Current source}

The Rogowski coils were designed to measure currents below $1 \mathrm{kA}$. The minimum necessary calibration current was therefore chosen as this value, i.e. $1 \mathrm{kA}$. As mentioned at the end of section 3, this current has to be driven at frequencies up to $1 \mathrm{kHz}$. To achieve this, the current carrier, in terms of the electronics circuit mainly acting as an inductance, is connected to capacitors to form a resonance circuit. This resonance circuit is then driven by a powerful AC-source. A transformer is utilized for impedance matching.

Fig. 5 shows the result of a simulation of this electrical circuit. The conductor employed was one of the conductors of the circumferential four conductor system, the capacitances were $15 \mu \mathrm{F}, 20 \mu \mathrm{F}, 30 \mu \mathrm{F}$, $40 \mu \mathrm{F}, 60 \mu \mathrm{F}, 100 \mu \mathrm{F}, 180 \mu \mathrm{F}, 420 \mu \mathrm{F}$ and $1680 \mu \mathrm{F}$, and the transformer ratio was 8 . In the resonance maxima the achieved current is above the targeted $1 \mathrm{kA}$.

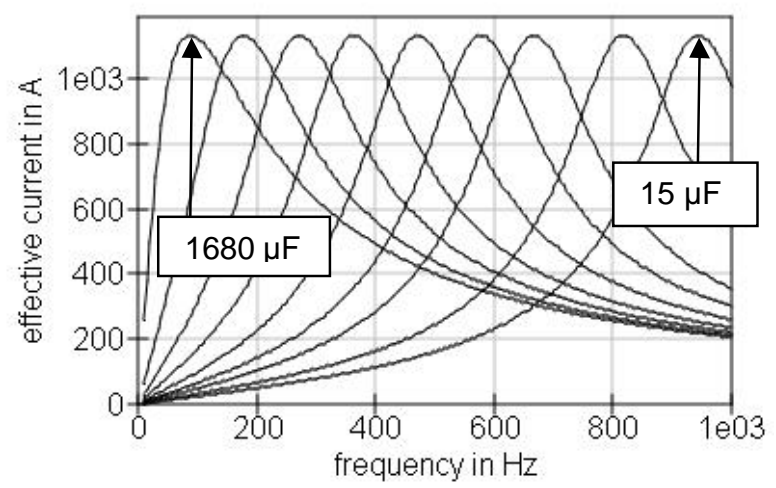

Fig. 5. Simulated calibration current driven through one of the conductors of the circumferential four conductor system.

However, the circuit needs further improvement since the internal resistances of several components and the transformer losses have not yet been incorporated into the simulation. Furthermore, the voltages over the capacitors need to be lowered to tolerable values. To achieve this, a second transformer for independent matching of the AC-source and the capacitances to the load is envisioned.

\section{Conclusions}

Low frequency calibration factors of the Rogowski coils mounted in W7-X could be calculated. High frequency calibration factors are planned to be provided by a staged approach with different conductors inside the plasma vessel and a multi-frequency AC-source.

\section{Acknowledgments}

The authors wish to thank the whole W7-X team for providing the framework that made the work presented here possible.

This work has been carried out within the framework of the EUROfusion Consortium and has received funding from the Euratom research and training programme 2014-2018 under grant agreement number 633053. The views and opinions expressed herein do not necessarily reflect those of the European Commission.

\section{References}

[1] M. Endler et al., Engineering design for the magnetic diagnostics of Wendelstein 7-X, Fusion Engineering and Design 100 (2015) 468-494.

[2] C. Beidler et al., Physics and Engineering Design for Wendelstein VII-X, Fusion Technol., 17 (1990) 148-168.

[3] H.-S. Bosch et al., Technical challenges in the construction of the steady-state stellarator Wendelstein 7-X, Nucl. Fusion 53 (2013) 126001.

[4] T. Klinger et al., Performance and properties of the first plasmas of Wendelstein 7-X, Plasma Phys. Control. Fusion 59 (2017) 014018.

[5] K. Rahbarnia et al., Commissioning of the magnetic diagnostics during the first operation phase at Wendelstein 7-X, Proc. 43rd EPS Conf. on Plasma Phys., Leuven, 4-8 July 2016, ECA Vol. 40A (2016) P4.011

[6] H. Thomsen et al., Status and prospects of the MHD diagnostics at Wendelstein 7-X stellarator, Proc. 43rd EPS Conf. on Plasma Phys., Leuven, 4-8 July 2016, ECA Vol. 40A (2016) P4.010 